\title{
DIVERSIDAD GENERICA DE HORMIGAS (HYMENOPTERA: FORMICIDAE) EN AMBIENTES DE BOSQUE SECO DE LOS MONTES DE MARIA, SUCRE, COLOMBIA
}

\section{GENERIC DIVERSITY OF ANTS (HYMENOPTERA: FORMICIDAE) IN DRY FOREST ENVIROMENTS OF MONTES DE MARIA, SUCRE, COLOMBIA}

\author{
DIAZ, P. JORGE A. ${ }^{1 *}$. MOLANO, P. CARLOS E. ${ }^{1}$. GAVIRIA, B. JULIO C. ${ }^{1}$. \\ ${ }^{1}$ Programa de Biología, Universidad de Sucre, Colombia. \\ * Correspondencia: jarnal412@hotmail.com
}

Las hormigas son uno de los grupos de insectos con mayor diversidad específica y ecológica en las latitudes tropicales, cumplen funciones importantes en todos los ecosistemas y constituyen alrededor del $15 \%$ de la biomasa animal total (VILLAREAL et al., 2006). Estos organismos presentan especialización en sus hábitos alimenticios y una estrecha relación con especies vegetales en especial de las familias Caesalpinaceae, Fabaceae, Melastomataceae, Cecropiaceae y Rubiaceae; Algunas especies se alimentan solo de huevos de artrópodos 0 exclusivamente de otras hormigas (HOLLDOBLER y WILSON, 1990).

La familia Formicidae está representada actualmente en la región Neotropical por 15 subfamilias: Agroecomyrmecinae, Amblyoponinae, Cerapachyinae, Dolichoderinae, Ecitoninae, Ectatomminae, Formicinae, Heteroponerinae, Leptanilloidinae, Myrmicinae, Paraponerinae, Ponerinae, Proceratiinae, Pseudomyrmecinae y la recientemente descrita Martialinae, que fue descubierta por RABELING et al. (2008) en el Amazonas Brasileño. FERNANDEZ y SENDOYA (2004), FERNANDEZ y SHARKEY (2006), afirman que hay algo más de 11.500 especies de hormigas descritas en 21 subfamilias vivientes; para el Neotrópico hay registradas unas 3.100 especies y 120 géneros; en Colombia se han registrado 91 géneros y cerca de 1.000 especies (FERNÁNDEZ 2003 y FERNÁNDEZ y SENDOYA, 2004).

Debido a su posición geográfica y gran diversidad de ecosistemas Colombia representa una de las zonas más ricas en hormigas de la región Neotropical (BROWN, 1991 apud. GUERRERO, 2007); a pesar de esto, la fauna de hormigas en el departamento de Sucre ha sido poco estudiada y ha merecido escaso interés por parte de los investigadores. JIMÉNEZ et al. (2008) en el trabajo titulado "Sistemática, Biogeografía y Conservación de las Hormigas Cazadoras de Colombia" registran para Sucre la presencia de varias especies de las subfamilias Ectatomminae y Ponerinae; sin embargo actualmente, de manera específica para este departamento no existen investigaciones publicadas acerca de la 
mirmecofauna. Por tal razón, el objetivo de este trabajo es dar a conocer algunas de las subfamilias y géneros de hormigas que están presentes en la subregión Montes de María del departamento de Sucre, con el propósito de contribuir al conocimiento de este grupo de insectos a nivel Regional.

El estudio se realizó durante los meses de julio a septiembre de 2009 en los municipios de Colosó y Sincelejo ubicados en la subregión Montes de María, la cual se encuentra localizada en la zona noreste del departamento de Sucre y constituida (además de los municipios mencionados anteriormente) por los municipios de Chalán, Morroa y Ovejas. Esta subregión se caracteriza como una zona de bosque seco tropical, con un paisaje característico de montaña. La temperatura promedio es de $26,8^{\circ} \mathrm{C}$, la precipitación varía entre 1.000 y $1.200 \mathrm{~mm}$ al año; la humedad relativa es del $77 \%$; el régimen de lluvia es bimodal y al corto período de lluvias del primer semestre le sigue un breve período seco en los meses de junio y julio; en el segundo semestre se presenta la mayor cantidad de precipitación pluvial (CARSUCRE, 2007)

La colecta de los especímenes se realizó en la vereda La Esmeralda del municipio de Colosó $\left(09^{\circ} 30^{\prime} 678^{\prime \prime} \mathrm{N}\right.$ y $\left.75^{\circ} 21^{\prime} 314^{\prime \prime} \mathrm{W}\right)$, y en los predios de la Universidad de Sucre, sede Puerta Roja en Sincelejo (09 18'902" N y 75²3'221" W), implementando trampas de caída y captura manual con pinzas y pinceles sobre la hojarasca, troncos en descomposición, frutos caídos, corteza de los árboles, ramas huecas, entre otros. Para la identificación taxonómica se utilizaron las claves de BOLTON (2003), FERNÁNDEZ (2003) y FERNÁNDEZ y SHARKEY (2006).

Se recolectaron en total 24 géneros de hormigas distribuidos en 7 subfamilias. (Tabla 1).

El número de subfamilias encontradas corresponde al $46,6 \%$ de las presentes en la región Neotropical. Dentro de estas, la subfamilia Myrmicinae presentó la mayor cantidad de géneros, $50,0 \%$ del número total encontrados; Dolichoderinae y Ponerinae cada una 12,5\%; Formicinae y Ecitoninae 8,3\%; de otra forma Pseudomyrmecinae y Ectatomminae 4,2\%.

A excepción de los géneros Atta, Trachymyrmex y Dolichoderus, que fueron colectados solo en el municipio de Colosó, los demás géneros fueron encontrados en las dos estaciones de muestreo. De manera general, los géneros de hormigas asociadas al suelo que presentaron mayor abundancia fueron Pheidole, Solenopsis, Pachycondyla y Ectatomma. De otra forma, en relación con plantas, 
fueron más abundantes los géneros Cephalotes, Crematogaster, Azteca y Pseudomyrmex (Fig.1), los cuales se observaron con más frecuencia sobre especies vegetales como: Samanea saman (campano), Enterolobium cyclocarpum (orejero), Cecropia peltata (yarumo), Annona muricata (guanábana), Brownea macrophylla (arizal), Sterculia apetala (camajón), Prosopis juliflora (trupillo), Crescentia cujete (totumo) y Anacardium excelsum (caracolí).

Tabla 1: Subfamilias y géneros de hormigas reportados para los Montes de María

\begin{tabular}{|l|l|}
\hline \multicolumn{1}{|c|}{ SUBFAMILIA } & \multicolumn{1}{|c|}{ GÉNERO } \\
\hline \multirow{5}{*}{ Myrmicinae } & Acromyrmex (Mayr,1865) \\
& Atta (Fabricius,1804) \\
& Cephalotes (Latreille,1802) \\
& Crematogaster (Lund,1831) \\
& Pogonomyrmex (Mayr,1868) \\
& Pyramica (Roger,1862) \\
& Pheidole (Westwood,1839) \\
& Monomorium (Mayr,1855) \\
& Solenopsis (Westwood,1840) \\
& Trachymyrmex (Forel,1893) \\
& Strumigenys (Smith, 1860) \\
& Wasmannia (Forel,1893) \\
\hline Dolichoderinae & Azteca (Forel,1878) \\
& Dolichoderus (Lund,1831) \\
& Dorymyrmex (Mayr,1865) \\
\hline Ponerinae & Anochetus (Mayr,1861) \\
& Odontomachus (Latreille,1804) \\
& Pachycondyla (Smith, 1858) \\
\hline Formicinae & Camponotus (Mayr,1861) \\
& Paratrechina (Motschoulsky,1863) \\
\hline Ecitoninae & Eciton (Latreille,1804) \\
& Neivamyrmex (Borgmeier,1940) \\
\hline Pseudomyrmecinae & Pseudomyrmex (Lund,1831) \\
\hline Ectatomminae & Ectatomma (Smith, 1858) \\
\hline & \\
\hline
\end{tabular}

Dentro de los especímenes colectados han sido identificados hasta el momento a nivel de especie Monomorium faraonis (Linnaeus), Odontomachus bauri (Emery), Ectatomma ruidum (Roger) y Eciton burchelli (Westwood). Para varios 
de los géneros encontrados en este trabajo no existen claves para las especies de Colombia o su taxonomía es relativamente compleja (FERNANDEZ, com. pers. Septiembre 2009).

Por otra parte, de acuerdo con GUERRERO y OLIVERO (2007), se puede afirmar que las áreas relictuales de bosque del Caribe colombiano pueden estar albergando gran cantidad de especies de hormigas endémicas o de amplia distribución que están a la espera de ser descubiertas. De esta manera, estudios sobre la diversidad de hormigas en la subregión Montes de María y demás subregiones del departamento de Sucre revisten gran importancia.

Este estudio generalizado puede ser considerado el comienzo de importantes descubrimientos sobre la mirmecofauna del departamento de Sucre y puede ser útil para posteriores estudios relacionados con diversos aspectos como su ecología y conservación.

Por último, se presenta un esquema con la morfología general de una hormiga y las descripciones generales para cada una de las subfamilias reportadas hasta el momento para la subregión Montes de María.

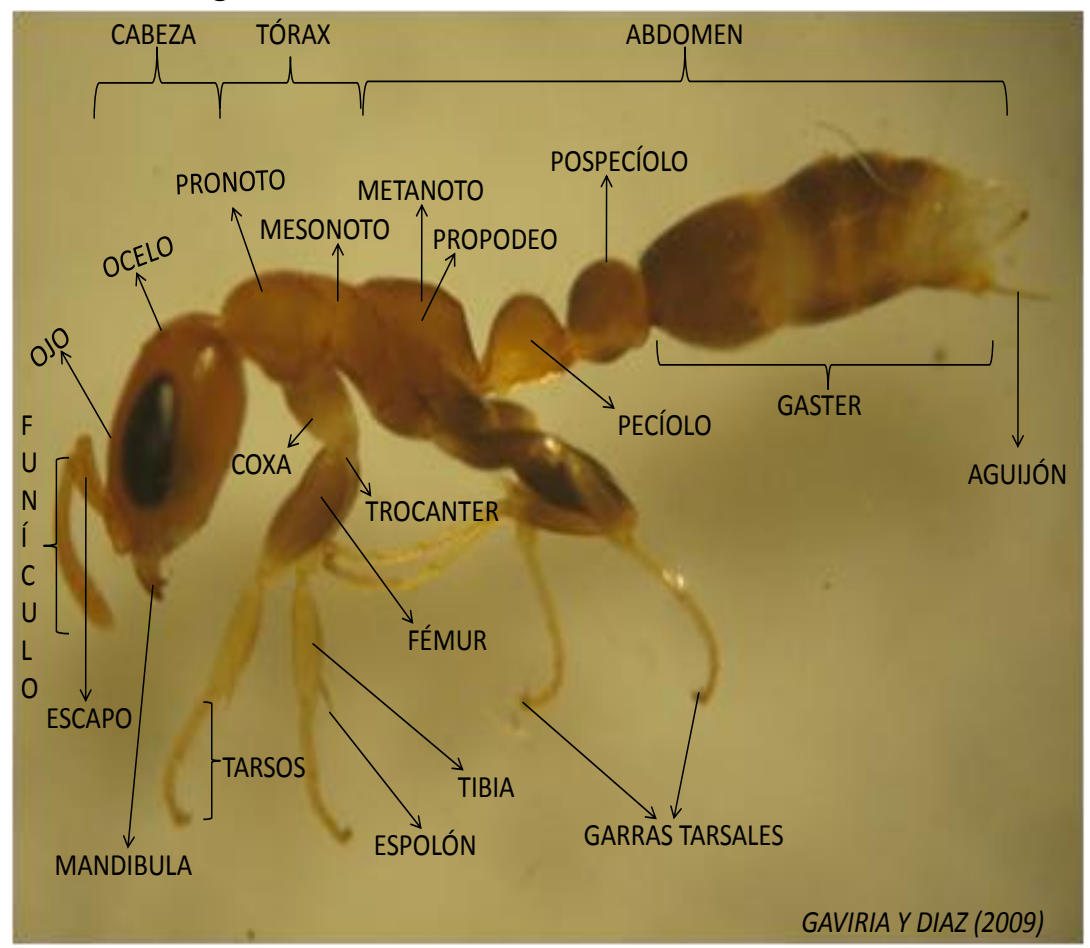

Figura 1. Morfología general de Pseudomyrmex (Lund, 1831). 
Descripción de cada subfamilia registrada en este trabajo (según FERNANDEZ 2003 y FERNÁNDEZ y SHARKEY 2006)

\section{Subfamilia Myrmicinae}

Clípeo se extiende medialmente entre las carenas frontales. Antenas de 4 a 12 segmentos, sin maza antenal o con maza de 1 a 4 segmentos. Lóbulos frontales presentes en la mayoría de géneros, algunas veces muy desarrollados. Ojos normalmente presentes, reducidos o ausentes en algunos grupos. Sutura promesonotal ausente, aunque puede haber una débil línea impresa. Lóbulos propodeales normalmente presentes, a veces reducidos. Pecíolo y pospecíolo presentes. Pigidio simple. Aguijón presente y funcional a reducido y no funcional.

\section{Subfamilia Formicinae}

Aguijón ausente. Ápice del gáster con un acidoporo circular a semicircular que sale del hipopigio y que usualmente está proyectado en forma tubular y bordeado por sedas, algunas veces cubierto por una proyección del pigidio. Clípeo amplio, normalmente no se extiende entre las carenas frontales. Antenas de 8 a 12 segmentos, raramente con maza antenal. Pecíolo normalmente visible en vistas lateral y dorsal (excepto en Paratrechina en vista dorsal). Espiráculos abdominales 4 en adelante normalmente ocultos.

\section{Subfamilia Ponerinae}

Clípeo generalmente amplio; inserciones antenales, con pocas excepciones tapadas por lóbulos frontales; ojos compuestos usualmente presentes, ocasionalmente atrofiados o ausentes; sutura promesonotal usualmente presente y flexible, a veces presente e inmóvil o ausente; peciolo de un segmento. Aguijón presente generalmente grande y bien desarrollado.

\section{Subfamilia Ecitoninae}

Clípeo reducido a una estrecha banda transversa de tal modo que las inserciones antenales se encuentran muy cerca de las mandíbulas. Inserciones antenales expuestas en vista frontal, lóbulos frontales ausentes. Antenas con 12 segmentos y un estrecho cuello uniendo el bulbo antenal con la base del escapo. Sutura promesonotal ausente, pronoto y mesonoto fusionados. Espiráculos propodeales situados muy arriba en los lados del propodeo. Glándula metatibial presente. Pecíolo de 1 (Cheliomyrmex) ó 2 segmentos (Eciton, Labidus, Neivamyrmex, Nomamyrmex). Aguijón bien desarrollado y funcional.

\section{Subfamilia Dolichoderinae}

Ojos generalmente presentes, rara vez vestigiales o ausentes, por lo general sin ocelos. Antenas de 8-12 segmentos. Carenas frontales presentes. Sutura 
promesonotal presente y flexible. Lóbulos propodeales ausentes. Pecíolo de un segmento y con fusión tergosternal. Pigidio pequeño. Hipopigio sin modificaciones. Acidoporo ausente. Aguijón vestigial o ausente, no visible sin disección.

\section{Subfamilia Pseudomyrmecinae}

Ojos notablemente desarrollados, ocupando cuando menos la mitad de los lados de la cabeza. Ocelos presentes. Antenas de 12 segmentos (11 en dos especies). Aguijón bien desarrollado. Las obreras de Pseudomyrmecinae pueden reconocerse por su aspecto alargado, ojos muy grandes, escapo corto, pospecíolo desarrollado y aguijón bien desarrollado. El pronoto y el mesonoto no presentan fusión, lo que implica que se pueden mover libremente entre sí. Pueden confundirse con obreras de Myrmicinae (por la posesión de pospecíolo), pero en estas los ojos son de tamaño moderado a reducido o ausente, el escapo normalmente es más largo, hay fusión entre el pronoto y mesonoto, y el pospecíolo no está tan desarrollado.

\section{Subfamilia Ectatomminae}

Cuerpo con dos segmentos aislados o reducidos (peciolo y pospecíolo) entre el mesosoma y el resto del abdomen. Cuerpo con un solo segmento aislado o reducido entre el mesosoma y el resto del abdomen. Orificio de la glándula metapleural en perfil longitudinal a oblicuo, rodeado debajo por un margen convexo de cutícula y dejando ubicado de manera dorsal el orificio (bula).

\section{Agradecimientos}

A Roberto Guerrero Flórez por su ayuda en la identificación taxonómica, dotación de material bibliográfico e importantes comentarios acerca del manuscrito. A Gustavo Manjarrez P., Gustavo Manjarrez G. y Giovanni Montes A. por su gran apoyo y motivación a nivel investigativo. A la familia Chamorro Quiroz y En especial a Luis Fernando por su colaboración en el trabajo de campo y hospitalidad en el municipio de Colosó. También expresamos gratitud a Saray Romero por su ayuda en el Laboratorio de Biología de la Universidad de Sucre.

\section{Referencias}

BOLTON, B. 2003. Synopsis and classification of Formicidae. The American Entomological Institute. Gainesville, FL.

CARSUCRE. 2007. Plan de Acción Trienal 2007-2009 Sincelejo-Sucre 
FERNÁNDEZ, F. 2003. Introducción a las hormigas de la región Neotropical. Instituto de Investigación de Recursos Biológicos Alexander von Humboldt, Bogotá, Colombia.

FERNÁNDEZ, F.; SENDOYA, S. 2004. List of Neotropical Ants (Himenóptera: Formicidae). Revista Biota Colombiana 5(1): 3-93

FERNÁNDEZ, F.; SHARKEY, M. J . 2006. Introducción a los Hymenoptera de la Región Neotropical. Sociedad Colombiana de Entomología, Bogotá D. C.

GUERRERO, R.; OLIVERO, D. 2007. Nuevo registros de hormigas del Caribe Colombiano, incluyendo claves taxonomicas para Acanthoponera, Heteroponera y Platythyrea. Revista Colombiana de Entomologia 33 (2):193-196

HOLLDOBLER, B.; WILSON, E.O. 1990. The Ants. Belknap press of Harvard University Press, Cambridge, Massachusetts, USA.

JIMÉNEZ, E.; FERNÁNDEZ, F; ARIAS, T.M.; LOZANO, F. H. 2008. Sistemática, biogeografía y conservación de las hormigas cazadoras de Colombia. Instituto de Investigación de Recursos Biológicos Alexander von Humboldt. Bogotá, Colombia.

RABELING, C.; BROWN, J.; VERHAAGH, M. 2008. Newly discovered sister lineage sheds light on early ant evolution. The National Academy of Sciences of the USA.

SANTAMARIA, C.; ARMBRECHT, J.; LACHAUD, J. P. 2009. Nest distribution and food preferences off Ectatomma Ruidum (Hymenoptera: Formicidae) in shaded and open cattle pastures of Colombia. Sociobiology 53(2b): 517-537

SNELLING, R.; FISHER, B.; WARD, P. 2007. Advances in ant systematics (Hymenoptera: Formicidae): homage to E. O. Wilson - 50 years of contributions. Memoirs of the American Entomological Institute.

VILLAREAL, H.; ALVAREZ, M.; CORDOBA, F.; FAGUA, G.; GAST, F.; MENDOZA, H.; OSPINA, M.; UMAÑA. 2006. Manual de métodos para el desarrollo de inventarios de biodiversidad. Instituto de Investigación de Recursos Biológicos Alexander Von Humboldt, Bogotá, Colombia. 